\title{
Handling Unknown User Arguments in Argumentative Dialogue Systems
}

\author{
Niklas Rach \\ Institute of Communications \\ Engineering, Ulm University \\ niklas.rach@uni-ulm.de
}

\author{
Wolfgang Minker \\ Institute of Communications \\ Engineering, Ulm University \\ wolfgang.minker@uni-ulm.de
}

\author{
Stefan Ultes \\ Department of Engineering, \\ University of Cambridge \\ su259@cam.ac.uk
}

\begin{abstract}
In this work we introduce a scheme to handle unknown user arguments in argumentative Dialogue Systems. We consider systems based on argument games and a graph representation of argument components and discuss how a component that is not present in the latter one can be included reasonably. The proposed approach also allows for a differentiation between invalid input and new arguments as well as a reasonable system response in the latter case.
\end{abstract}

Natural Language Understanding, Computational Argumentation, Dialogue Systems

\section{INTRODUCTION}

Argumentative Dialogue Systems aim for an interaction with human users by exchanging arguments via natural language in order to for example persuade or resolve conflicts. One of the main challenges in the implementation of suchlike systems (Yuan, 2011) lies in the Natural Language Understanding (NLU) of arguments, as the system has to identify the content of the user's utterance and the respective relation to other arguments to answer appropriately. This also includes a reasonable handling of content unknown to the system as the user's argument may not be present in the respective database. Thus, the system has to determine whether the user's input is a reasonable, but unfamiliar response and if so, how to appropriately react to it.

Within this work, we introduce an approach for handling unknown user arguments in argumentative Dialogue Systems based on techniques from the field of argument mining. It allows for a structural analysis of the user utterance and thus for an appropriate reaction of the system. We focus the discussion on systems that are based on argument games (Prakken, 2005) and discuss schemes to process the respective unknown input as well as to react reasonably to it. The proposed approaches extend the ones presented in Rach (2017).

The remainder of this paper is as follows: Section 2 introduces system components that are of importance for the discussion at hand, whereas the specific handling of unknown user input in the context of the complete NLU task at hand is discussed in Section 3. We close in
Section 4 with a conclusion and a discussion of future steps.

\section{ARGUMENTATIVE DIALOGUE SYSTEM}

In this section we discuss the overall framework and relevant components of an argumentative Dialogue System our approach is based on. Throughout this work we consider systems that model the interaction as a dialogue game for argumentation (or argument game) in which the participants play moves that are in line with a framework specific set of rules. Although this generally restricts the interaction, it also ensures a certain structure and reasonable system responses. Frameworks of this kind generally allow not just for the exchange of arguments but also for strategical moves like for example challenging the validity of an opponent's argument or to retract an own previous one. This enables each party to continue the interaction even if no immediate counter argument is available. As this is the case if an unknown argument is employed, systems of this type are suitable candidates for our problem at hand since they reduce the problem of dealing with an unknown user argument to the problem of identifying an appropriate response move.

Independently of the specific formalization (i.e. the respective set of rules), each system requires knowledge about existing arguments and their relation to each other. $A$ respective database thus has to encode the available argument components as well as the logical connections between them. These can be represented as a graph in which the nodes constitute the components and the edges the respective relations. 
Given a specific set of rules and a database of arguments, the problem of NLU in a suchlike system is divided into two tasks. The first one is to identify the type of move played by the user and the second one is to identify the component it refers to. This is illustrated in the following example, based on the argument game introduced by Prakken (2000) and the topic "marriage is an outdated institution"'. This specific game includes the following moves:

- Claim (opening the game).

- Argue (introducing a new argument component and the respective relation to previous components).

- Why (challenging the validity of an argument component).

- Concede and Retract (conceding to or retracting a previous argument).

Given the following system utterance and the respective user reply

\section{S1: Marriage is an outdated institution.}

U1: Marriage removes the transient and casual aspects of a monogamous relationship, thus giving a child a far more stable environment.

the NLU first has to classify the user utterance as argue move and secondly to determine the argument component in the database the user refers to. The second task can be facilitated by first identifying the aim of the move, i.e. the component the utterance refers to and is illustrated in Figure 1.

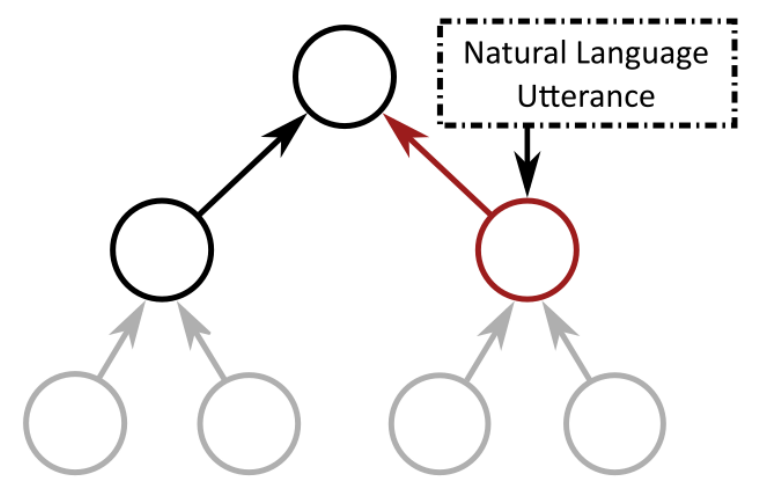

Figure 1: Mapping of the utterance to a component included in the database. The red node indicates the component the utterance is mapped to.

Consequently, in order to deal with unknown user input, the system first has to identify whether or not the utterance encodes a legal move in the respective game and if so, to determine the relation(s) of the freshly introduced component to others that are already included into the database.

\footnotetext{
${ }^{1}$ Material reproduced from www.iedebate.org with the permission of the International Debating Education Association. Copyrightc 2005 International Debate Education Association. All Rights Reserved
}

\section{HANDLING UNKNOWN USER INPUT}

In this section we discuss components of the NLU that may be employed to solve the above discussed tasks and the extensions that are required in order to deal with unknown content.

The database or argument structure of the system encodes all the knowledge the system has about the discussed topic. Thus, the user's input has to be mapped (in the case of a familiar argument or an alternative move) or integrated (in case of a new argument) into this database to allow an appropriate response of the system. Consequently, the natural language utterance of the user has to be (automatically) processed into a form that fits this representation. In this process, different questions have to be addressed:

- Q1: Is the user's utterance a legal move in the current state of the game? (Task 1 in the previous Section)

- Q2: If so, is the respective component known and allowed (as target or component) in the current state of the game? (Task 2 in the previous Section)

- Q3: If it is not, is the new content valid in the current state of the game?

The first question refers to the identification of moves. If the user input encodes an invalid move or nonsense statement, the system can reject it and ask for an alternative formulation. This subtask may be addressed by state of the art NLU tools as for example the ones compared in Braun (2017).

The second question includes the mapping of the utterance to a known component in the database (if additional content is introduced to the game) or an identification of the respective component played earlier (if the actual utterance refers to it). This may be achieved by either employing semantic similarity measures as for example the one introduced in $\mathrm{Li}$ (2006) or word embedding.

The third question is difficult to answer because the system has to determine the validity of new components. As the utterance was at this point already recognized to contain an argument, the remaining question is its relation to the ones encoded in the database. The automatic analysis and extraction of argument structures, including the relations between different components is a major task in the field of argument mining. Recently, argument mining techniques were also employed in order to build the earlier discussed argument database for an argumentative Dialogue System from a natural language source (Rach, 2018). We thus propose an approach that combines both aspects by training the architectures employed for the recognition of relations on the data encoded in the argument database. Depending on the size of this database, the argument components on a 
certain topic are not enough data to form a complete training corpus. However, if the representation of arguments in the database matches the argument mining scheme, existing corpora like the one introduced in Stab (2014) may be employed to pre-train the architecture. The latter one may then be utilized in an ongoing dialogue in order to determine how a freshly introduced argument component is related to the existing ones. It is worth noting that this approach is not limited on finding an appropriate response to unfamiliar arguments but also enables the system to learn the new content by including it into the database. Consequently, the unfamiliar argument can be utilized by the system itself in later dialogues. Similar to humans, the system is thus able to remember arguments even if they were not included in the database in the first place.

We illustrate the complete procedure for a new argument component by extending the above discussed example with the topic "marriage is an outdated institution". In the following dialogue, the user responds to the systems initial claim (S1) with an unfamiliar argument (U1).

\section{S1: Marriage is an outdated institution.}

\section{U1: Marriage promotes a better way to raise children.}

The system first detects the type of move (Q1) - in this case argue. As an argue move is a valid response to the initial claim, the system now matches the utterance with known argument components in the database (Q2) by for example computing the semantic similarity between the utterance and the known components. As a respective component is not included in the database (i.e. each similarity score is below a certain threshold), the system now investigates whether or not the content is related to the content of the initial claim by processing it through the respective argument mining architecture (Q3). As the user utterance directly addresses the claim, it is a legal response and the system accepts the new component as well as the respective move and selects an appropriate answer. In the herein considered argument game, possible responses are to challenge the new component (S2.1) or concede to it (S2.2).

\section{S2.1: Why do you think that?}

\section{S2.2: I agree with you.}

In a later state of the dialogue, the system could also change the topic by introducing an own counter argument on an earlier (familiar) one of the

\footnotetext{
${ }^{2}$ Material reproduced from www.iedebate.org with the permission of the International Debating Education Association. Copyrightc 2005 International Debate Education Association. All Rights Reserved
}

opponent. In each case, the system extends its own database by including the new component.

\section{CONCLUSION}

We have presented and approach for dealing with unknown user input in argumentative Dialogue Systems. The introduced scheme stems on the formalization of the interaction as an argument game in order to provide a reasonable answer and employs argument mining techniques to include the user utterance into the database of arguments. In doing so, the system is not just able to react to an unfamiliar argument but also to utilize it in future dialogues. It should be noted that systems that do not rely on argument games like the one introduced in Rakshit (2017) may also employ the discussed scheme in order to include the additional information. However, the question of how to react to the new content has to be addressed in a different way in a suchlike system.

In our perspective the general complexity of argumentative dialogue makes the ability of including unknown arguments into the own database crucial for future systems of the respective kind. Many dialogical tasks as for example deliberation depend on the exchange of information and thus on the ability of understanding a new argument if they are addressed in human computer interaction. In addition, competitive tasks such as debating also require a handling of unknown content in order to be flexible and realistic as it is not reasonable to assume that all arguments known to the user are also known to the system. As a consequence, concepts for handling new content on the level of NLU and the Dialogue Manager are essential in the development of argumentative Dialogue Systems.

Future research directions will thus focus on the implementation of the respective system components and will address the herein described $\mathrm{NLU}$ as well as strategical aspects, including the investigation of different strategies to react to unfamiliar arguments.

Acknowledgements: This work is part of a project that is funded by the Deutsche

Forschungsgemeinschaft (DFG, German Research Foundation) - 376696351.

\section{REFERENCES}

Braun, D., Hernandez-Mendez, A., Matthes, F., \& Langen, M. (2017). Evaluating natural language understanding services for conversational question answering systems. In Proceedings of the 18th Annual SIGdial Meeting on Discourse and Dialogue (pp. 174-185). 
Li, Y., McLean, D., Bandar, Z. A., O'shea, J. D., \& Crockett, K. (2006). Sentence similarity based on semantic nets and corpus statistics. IEEE transactions on knowledge and data engineering, 18(8), 1138-1150.

Prakken, H. (2005) Coherence and flexibility in dialogue games for argumentation. Journal of logic and computation 15(6):1009-1040.

Prakken, H. (2000, September). On dialogue systems with speech acts, arguments, and counterarguments. In European Workshop on Logics in Artificial Intelligence (pp. 224-238). Springer, Berlin, Heidelberg.

Rach, N., Minker, W., \& Ultes, S. (2017). Towards an Argumentative Dialogue System. In Proceedings of 17th Workshop on Computational Models of Natural Argument (CMNA'17)

Rach, N., Minker, W., \& Ultes, S. (2018). Utilizing Argument Mining Techniques for Argumentative Dialogue Systems. Presented at International Workshop on Spoken Dialog System Technology (IWSDS) 2018, Singapore

Rakshit, G., Bowden, K. K., Reed, L., Misra, A., \& Walker, M. (2017). Debbie, the Debate Bot of the Future. arXiv preprint arXiv:1709.03167.

Stab, C., \& Gurevych, I. (2014). Annotating argument components and relations in persuasive essays. In Proceedings of COLING 2014, the 25th International Conference on Computational Linguistics: Technical Papers (pp. 1501-1510).

Yuan, T., Moore, D., Reed, C., Ravenscroft, A., \& Maudet, N. (2011). Informal logic dialogue games in human-computer dialogue. The Knowledge Engineering Review, 26(2), 159-174. 